\title{
Muséologies
}

Les cahiers d'études supérieures

\section{Découvrir en famille les oeuvres de Pierre Soulages après une visite scolaire}

\section{Aude Joly et Marie-Sylvie Poli}

Volume 7, numéro 1, 2014

Le dialogue dans les musées d’art contemporain

URI : https://id.erudit.org/iderudit/1026650ar

DOI : https://doi.org/10.7202/1026650ar

Aller au sommaire du numéro

Éditeur(s)

Association Québécoise de Promotion des Recherches Étudiantes en

Muséologie (AQPREM)

ISSN

1718-5181 (imprimé)

1929-7815 (numérique)

Découvrir la revue

Citer cet article

Joly, A. \& Poli, M.-S. (2014). Découvrir en famille les oeuvres de Pierre Soulages après une visite scolaire. Muséologies, 7(1), 123-139.

https://doi.org/10.7202/1026650ar

Tous droits réservés (C Association Québécoise de Promotion des Recherches Étudiantes en Muséologie (AQPREM), 2014
Ce document est protégé par la loi sur le droit d'auteur. L'utilisation des services d'Érudit (y compris la reproduction) est assujettie à sa politique d'utilisation que vous pouvez consulter en ligne.

https://apropos.erudit.org/fr/usagers/politique-dutilisation/ 
Article cinq

\section{Découvrir en famille les œuvres de} Pierre Soulages après une visite scolaire Aude Joly et Marie-Sylvie Poli 
Aude Joly est titulaire d'une licence en histoire de l'art. En 2009, elle a complété une maîtrise en muséologie à l'Université du Québec à Montréal. Elle a depuis ouvré en tant que médiatrice et chargée de projets pour le musée des Augustins de Toulouse et le musée Fabre de Montpellier. Depuis 2010, elle travaille à une thèse de doctorat en muséologie à l'Université d'Avignon et des Pays de Vaucluse, sous la direction de Marie-Sylvie Poli. Ses recherches visent à mieux connaître les comportements du public familial dans les musées de beaux-arts. aude.joly84@gmail.com

Professeure à l'Université d'Avignon et des Pays de Vaucluse (France), chercheure en muséologie au Centre Norbert Elias - Équipe culture et communication, Marie-Sylvie Poli est codirectrice de la Structure fédérative de recherche Agorantic "Culture, patrimoines, sociétés numériques ". Ses travaux portent sur les effets de la parole et de la langue dans les dispositifs de médiation culturelle, plus spécialement dans les musées et les expositions. Deux questions l'intéressent particulièrement: Comment " parle " l'exposition? Comment le visiteur s'engage-t-il dans le " dialogue " qui lui est proposé par le musée? marie-sylvie.poli@univ-avignon.fr 
On assiste depuis quelques années à une sectorisation du discours de communication des musées, qui, de plus en plus, s'adressent à une catégorie relativement récente de visiteurs: les familles. Les termes "public famille ", visite " en famille ", " pour les familles " se déploient en effet de manière quasi généralisée sur les sites Internet et les programmes d'activités culturelles des musées. En s'adressant au "public famille ", le musée met l'accent sur l'aspect social, communicationnel et affectif de la visite. Considérer la famille comme public revient à prendre en compte les visiteurs dans leur relation à l'œuvre et à l'exposition, mais aussi dans leur relation à l'autre: à celui et ceux qui l'accompagnent dans son expérience de visite. Qu'en est-il de l'expérience de visite en famille et, plus particulièrement, de la visite d'un musée d'art? Que se passe-t-il quand un adulte et un enfant découvrent et observent ensemble une œuvre?

La recherche en muséologie s'intéresse depuis une trentaine d'années à la famille en tant que public. Les recherches dans le domaine se sont principalement développées dans des musées à caractère scientifique. Bien que moins nombreuses, les recherches sur les familles au sein des musées d'art nous donnent néanmoins des indications précieuses, nous renseignant sur le profil socioéconomique du public familial ${ }^{1}$ ainsi que sur ses motivations et ses logiques de visite $^{2}$. En ce qui concerne la fréquentation des musées d'art français, on sait notamment que les familles qui les fréquentent présentent un profil que l'on peut qualifier d'élitiste ${ }^{3}$ et que celles-ci le visitent majoritairement dans

\section{BEAUMONT, Éla et Pat STERRY. « Methods for} Studying Family Visitors in Art Museums: A Cross-disciplinary Review of Current Research ». Museum Management and Curatorship, vol. 21, no 3, 2006, p. 222- 239; et EIDELMAN,Jacqueline et Anne JONCHERY. « Sociologie de la démocratisation des musées ". In. RASSE, Paul et Yves GIRAUD (dir.). Hermes, no 61, Les musées au prisme de la communication, 2011, p. 52-60.

2 JONCHERY, Anne. Quand la famille vient au musée: des pratiques de visites aux logiques culturelles. Thèse de doctorat en muséologie, Paris, Muséum national d'histoire naturelle, 2005. 3 EIDELMAN et JONCHERY, "Sociologie de la démocratisation des musées ", op. cit., p. 52-60.

4 JONCHERY, Quand la famille vient au musée..., op. cit.

5 EIDELMAN et JONCHERY, « Sociologie de la démocratisation des musées ", op. cit., p. 52-60; et MIRONER, Lucien. une logique de transmission éducative et de distinction culturelle ${ }^{4}$. Par ailleurs, les études de publics révèlent que la fréquentation familiale des musées d'art français reste non seulement marginale, mais aussi stagnante ${ }^{5}$. Les études portant sur les familles dans les musées s'intéressent aussi régulièrement aux médiations qui leur sont spécifiquement destinées ${ }^{6}$. Malgré toutes ces informations, " nombreux sont les aspects des visites familiales dans les musées d'art qui restent inexplorés ${ }^{7}$ ", notamment ceux relatifs à l'expérience de visite.

Dans cet article, nous proposons de pousser la porte du musée pour interroger la manière dont parents et enfants interagissent face aux œuvres lorsqu'ils visitent ensemble et de manière libre un musée d'art. Plus particulièrement, il s'agira de comprendre l'impact d'une visite scolaire suivie au préalable par l'enfant sur l'attitude et le comportement de ses parents lors d'une visite en famille dans les salles du musée Fabre de Montpellier dédiées à l'œuvre de l'artiste contemporain Pierre Soulages. En quoi le fait que l'enfant ait participé à une visite scolaire influence-t-il les conversations et les relations des adultes et de leurs enfants face à de l'art abstrait?

\section{La visite en famille: un modèle de transmission verticale et descendante}

Le public famille est considéré comme un groupe de visiteurs dont les interactions diffèrent des autres publics en groupe. Pour Jean-Pierre Cordier ${ }^{8}$, la visite familiale relève

Cent musées à la rencontre du public. Cabestany: France Édition, 2001

6 DUCLOS, Amandine, Franck PHILIPPEAUX et Marie-Sylvie POLI. "De l'utilité du " texte enfant " au musée ". In. POLI, Marie-Sylvie (dir.). La Lettre de l'Ocim. Numéro spécial 132, "Le texte dans l'exposition ", 2010, p. 28-33; et GOTTEDIENER, Hanna et Jean-Christophe VILATTE. « Impact of a Game Booklet on Family Visit to an Art Exhibition ". Empirical Studies of the Arts, vol. 19, no 2, 2001, p. 167-176. 7 Traduction libre de " many aspects of family visits to art museums remain relatively unexplored ". BEAUMONT et STERRY, "Methods for Studying Family Visitors... ", op. cit., p. 222.

8 CORDIER, Jean-Pierre. « La transmission familiale au musée ». In. ALLARD, Michel et Bernard LEFEBVRE (dir.). Le musée, un lieu éducatif. Montréal, Musée d'art contemporain, 1997, p. 188. 
d'un travail collectif fondé sur une relation d'interdépendance entre plusieurs partenaires de générations différentes ayant une histoire commune et associés dans un projet de transmission. Qu'il soit conscient ou non, le processus de transmission est indissociable de la pratique de visite en famille, moment particulièrement propice à des échanges entre générations centrés sur la transmission d'éléments provenant d'un double héritage: celui du milieu familial et celui représenté par les collections du musée. La transmission intergénérationnelle, notamment dans le domaine de la culture, est largement considérée comme un processus vertical et descendant (c'est-à-dire des parents vers l'enfant). En partant de ce schéma vertical, «les transmissions familiales peuvent recourir à deux mécanismes: d'une part, l'éducation volontaire, plus largement nommée "inculcation" $[. .$.$] de l'autre, l'imprégnation, par expo-$ sition de l'enfant aux exemples parentaux ${ }^{9}$ ”. Ainsi, dans ce modèle de transmission, le rapport de l'adulte à l'enfant se rapproche d'une relation correspondant à un rapport « maître / une "pression éducative " par l'adulte, qui se sent « investi d'une mission de transmission pédagogique $^{10}$ ". François De Singly reconnaît d'ailleurs avant tout à l'adulte le rôle d'accompagnateur, considérant qu'il " est dans la situation avec surtout son identité statutaire $[. .$. en tant que parent, alors que l'autre [l'enfant] vit cela avec son identité personnelle ${ }^{11}$ ».

9 OCTOBRE, Sylvie et Yves JAUNEAU. « La transmission culturelle: tels parents, tels enfants? ”. La revue française de sociologie, vol. 49, no 4, 2008, p. 701.

10 DEBENEDETTI, Stéphane. "Visites occasionnelles du musée d'art et confort de visite: le rôle des compagnons ". In. DONNAT, Olivier et Paul TOLILA (dir.). Le(s) public(s) de la culture. Paris: Presses de Science Po, 2003, p. 278.

11 DE SINGLY, François. « La famille individualiste face aux pratiques culturelles ". In DONNAT et TOLILA (dir.), Le(s) public(s) de la culture, op. cit., p. 52-53.

\section{La visite scolaire comme une variable pour réinterroger ce modèle}

Afin de réinterroger ce modèle de transmission reconnu comme majoritaire dans les groupes familiaux en visite, nous avons choisi d'introduire, pour cette étude, la variable " visite scolaire ». En partant des constats selon lesquels la grande majorité des visites effectuées par les jeunes de moins de 18 ans se font dans le cadre scolaire, que les enfants français scolarisés profitent d'une forte diffusion de la pratique muséale par le biais de l'école ${ }^{12}$ et que les visites scolaires sont surreprésentées dans les musées d'art ${ }^{13}$, nous interrogeons dans cette étude le rôle que jouent ces visites scolaires à l'occasion de visites ultérieures en famille. En invitant des enfants qui ont participé à une visite scolaire à revenir visiter le musée avec leurs parents, cette recherche interroge le rôle de l'enfant dans la visite en famille, mais aussi l'impact que la pratique du musée dans le cadre scolaire peut avoir sur les attitudes, les conversations et les modes de communication/transmission des familles qui visitent un musée d'art. La visite scolaire constitue-t-elle une passerelle entre le musée et la famille?

Nous pouvons poser, comme hypothèses de départ, que:

- lors d'une visite en famille, la pratique scolaire du musée aurait un impact sur la construction et / ou l'évolution des images que les parents se font du musée d'art et des œuvres d'un artiste contemporain;

- l'enfant, en passant du statut d'élève à celui d'enfant visiteur, pourrait endosser un rôle de guide, voire de médiateur du musée et des œuvres d'art auprès de ses parents.

12 "Le musée est [...] l'équipement culturel qui bénéficie le plus des efforts de sensibilisation scolaire (les trois quarts des 6-14 ans y sont allés dans le cadre scolaire, contre $60 \%$ environ pour les châteaux, $50 \%$ environ pour les spectacles et moins de $30 \%$ pour les bibliothèques) ". OCTOBRE, Sylvie. "Connaître les populations et les publics". In. EIDELMAN, Jacqueline, Mélanie ROUSTAN et Bernadette GOLDSTEIN (dir.). La place des publics. De l'usage des études et recherches par les musées. Paris: La Documentation française, coll. «Musées-mondes ", 2008, p. 144.

13 EIDELMAN, Jacqueline, Anne JONCHERY et Lucile ZIZI. Musées et publics: bilan d'une décennie (2002-2011). Paris: Ministère de la Culture et de la Communication, juin 2012, p. 7. 


\section{Méthodologie $^{14}$}

\section{Une visite des salles de l'œuvre de Soulages réalisée dans le cadre scolaire}

Pour répondre à ces questions, une enquête de terrain est actuellement réalisée au musée Fabre de Montpellier. Plus précisément, l'espace choisi se concentre sur la partie moderne et contemporaine de l'exposition permanente renfermant les salles consacrées à l'œuvre de Pierre Soulages ${ }^{15}$. Dans un premier temps, trois classes de la ville de Montpellier participantes à l'étude (CE1, CE2 et CM $\left.2{ }^{16}\right)$ ont suivi une visite des espaces Soulages animée par une médiatrice du musée ${ }^{17}$. La visite, intitulée "Le noir-lumière de Pierre Soulages ", dure environ une heure. Elle propose aux élèves de découvrir de manière sensible le travail du peintre ainsi que la mise en exposition que le musée Fabre lui a consacrée, cette dernière ayant en effet été pensée et conçue en collaboration avec l'artiste pour accueillir sa donation faite en 2005 à l'occasion de la réouverture du musée. À la fin de la visite, un temps personnel est proposé aux jeunes visiteurs. Munis d'une feuille et d'un crayon, ils sont invités à se placer devant leur œuvre préférée pour l'observer plus attentivement, écrire de manière spontanée les mots qu'elle leur inspire et la dessiner. De retour en classe, les élèves sont informés plus précisément de l'étude en cours et sont invités dans ce cadre (et s'ils le souhaitent) à revenir visiter gratuitement ces espaces avec leur(s) parent(s) dans le mois qui suit.

\section{La visite en famille enregistrée}

La méthodologie consiste à enregistrer à l'aide d'un micro-cravate les conversations des familles alors qu'elles visitent à leur rythme et de manière autonome les salles consacrées à l'œuvre de Soulages. Il est important que la visite se fasse de manière libre. Les membres

14 Nous tenons à remercier la direction et le service des publics du musée Fabre de Montpellier Agglomération d'avoir rendu possible la réalisation de cette étude.

15 Voir le site du musée Fabre: <http://museefabre. montpellier-agglo.com/content/view/salle/215> (consulté en janvier 2013)

16 Dans le système scolaire français, $\mathrm{CE}=$ cours élémentaire (le chiffre représentant la première ou la deuxième année) CM = cours moyen

17 La même médiatrice accueille et guide les classes partici- de la famille évoluent seuls et sont maîtres de la durée, du rythme de leur visite et de leurs déplacements. Par ailleurs, les familles ne disposent d'aucune autre forme de médiation que la mise en exposition elle-même, dont les textes présents dans l'espace, à savoir les cartels et les fiches de salles ${ }^{18}$. La visite est suivie d'un entretien semi-directif portant sur leurs habitudes de visites en famille et sur l'expérience qu'ils viennent de vivre ensemble.

Le présent article se concentre sur l'analyse des entretiens menés en fin de visite avec huit familles. L'échantillon n'est volontairement pas un reflet du public familial du musée Fabre. Il s'agit de familles qui ne sont pas éloignées de la pratique muséale, mais dont les habitudes de visite sont différentes, allant de la faible fréquentation à la fréquentation assidue des musées. Par ailleurs, elles ont toutes la particularité de visiter le musée Fabre pour la première fois en famille.

Les phénomènes et les processus présentés dans cet article sont issus de l'analyse des conversations et des entretiens menés avec un nombre réduit de familles. Il ne s'agit pas ici de présenter des résultats pouvant prétendre à une généralisation ou à une représentativité sur une échelle quantitative. C'est pourquoi les tendances qui se dégagent devront pour l'instant être considérées comme des hypothèses de travail.

Les questions principales soulevées sont les suivantes: comment une visite scolaire vécue par l'enfant en amont d'une visite en famille peut-elle influer sur l'expérience de visite de son / ses parents, en matière de représentations personnelles que ce dernier se fait de l'œuvre d'un artiste contemporain et du musée, mais aussi de son expérience de visite?

pantes. Ses propos sont enregistrés et transcrits. 18 Les fiches de salles sont consultables sur le site Internet du musée Fabre: «Le renouveau de la peinture à l'époque contemporaine ". Fiche de la salle $46:<$ http://museefabre. montpellier-agglo.com/pdf.php/?filePath=var/storage/original/application/7aabd341624bdef982454901819b963f. pdf>; et fiche de la salle $47:<$ http://museefabre.montpellier-agglo.com/pdf.php/?filePath=var/storage/original/application/33bb5b7716eee3ce8ea19fb54733eb6c.pdf> (sites consultés en janvier 2013). 
Observer en famille des œuvres après une visite scolaire: quel impact sur les attitudes des parents?

\section{Visiter une exposition d'art abstrait: les freins à la visite en famille}

Sur les huit familles rencontrées, six s'accordent pour dire qu'elles ne seraient pas venues d'ellesmêmes visiter avec leur enfant les espaces du musée dédiés à l'art abstrait de Pierre Soulages. Tout du moins, elles se seraient contentées de les traverser rapidement, " par curiosité ». Il était donc intéressant de les interroger sur ce qui les retenait de visiter en famille un espace dédié à l'art abstrait.

\section{Le goût des parents}

La première raison invoquée trouve sa source dans le goût des parents, puisque quatre parents expliquent en effet ne pas être touchés ou attirés par l'art abstrait et contemporain en général ou par l'œuvre de Soulages en particulier. Ils n'auraient, par conséquent, pas fait la démarche de se rendre dans ces espaces du musée avec l'importance que semblent avoir les goûts parentaux sur les choix de visite dans le cadre familial:

$\mathbf{M}^{19}$ : Moi j'avais vu Soulages il y a deux ans $[. .$.$] J'avais gardé une très mauvaise$ impression de visite.

I: Quand vous aviez visité, vous vous étiez dit « je vais montrer ça à $H$. »?

M: Ah non pas du tout, ah non vraiment pas! Par contre d'autres choses oui, enfin, c'est un réflexe de mère, quand je vois quelque chose de beau, puis y'a même certains trucs, que ce soit la musique, la peinture, je me dis, oui, il faudra que je revienne avec les enfants (mère de H., dix ans).

On retrouve un phénomène évoqué par Anne Jonchery, qui a montré comment la visite familiale d'un musée d'art semblait moins intégrer le plaisir de l'enfant pour donner la part belle à l'intérêt de l'adulte. Dans les musées d'art,

dit-elle, l'intérêt personnel de l'adulte comme motivation à la visite est bien plus représenté ${ }^{20}$. Au-delà des goûts du parent, le choix d'une visite en famille semble aussi reposer sur la représentation que les adultes ont des œuvres exposées, ici, en l'occurrence, des œuvres abstraites d'un artiste contemporain.

\section{L'image d'un art difficilement accessible en famille}

Une deuxième raison est avancée de manière très précise par deux mères. Elles connaissaient préalablement le travail du peintre et considéraient ses œuvres abstraites comme difficilement accessibles pour leur enfant. La démarche artistique de Soulages serait, d'après elles, davantage parlante pour des adultes: "C'est un art, comment dire, plus abstrait, plus intellectualisé. C'est-à-dire que les adultes doivent effectivement réfléchir sur la lumière, sur le relief, tout ça. Ça me semble moi très difficile d'accès aux enfants ", et par là même d'autant plus difficile pour des adultes d'y être confrontés en présence d'enfants : "Les peintures abstraites c'est pas trop fait pour les enfants, je pense surtout que c'est difficile d'accès pour les adultes, et qu'on a du mal a y amener rapidement, naturellement les enfants " (mère de J., dix ans). Cette dernière remarque se retrouve dans le discours de plusieurs des parents. En effet, ces derniers évoquent la difficulté qu'ils auraient eue à partager avec leur enfant ce type d'œuvres:

Ce n'est pas que ce n'est pas accessible, parce que je suis sûre qu'elle y est sensible et qu'elle est réceptive. C'est plus moi, je pense qu'on s'y serait promenées, mais je n'aurais pas vraiment su quoi lui dire. Alors qu'avec l'art figuratif, on ramène à des choses du quotidien, à leur environnement proche, on peut jouer sur la mine que fait un personnage... Mais l'abstrait, c'est assez difficile. À part dire quelle couleur, essayer de retrouver des formes... Moi ça me laisse plus sèche dans le discours en tout cas. En tant que maman, j'aurais eu du mal à engager la conversation (mère de A., huit ans). 
Si la possibilité leur en avait été donnée, les choix de visite de ces six familles se seraient donc portés sur des salles considérées comme plus " classiques ", présentant des œuvres figuratives, considérées comme plus accessibles pour leur enfant. "J'imaginais que quand on avait cet âge-là, on était plus sensible, par exemple, à des tableaux de Berthe Morisot, ou devant une sculpture, ou des tableaux du $\mathrm{XIX}^{\mathrm{e}},[\ldots]$ plus lumineux, disons plus colorés, plus accessibles " (mère de J., dix ans). "Sincèrement [...], on m'aurait proposé entre impressionnistes et Soulages, je serais plus allée vers l'impressionnisme, vers quelque chose de plus, qui me semble plus facile à regarder " (mère de L., sept ans).

Les propos de ces familles concernant les freins à la visite d'espaces d'art abstrait illustrent ce que Sylvie Octobre et Yves Jauneau appellent la reproduction négative. Ces auteurs expliquent que c'est « la posture de retrait qui se transmet le mieux $"^{21}$. Toutefois, il semblerait que la visite scolaire dédiée à un artiste abstrait pourrait être le point de départ d'un changement d'opinion pour certains des parents. Le crédit qu'ils portent aux choix de l'école et des enseignants les incitant, pour certains, à revoir leur première position.

\section{Le crédit porté à l'école: de la visite scolaire...}

La visite scolaire en elle-même paraît importante dans le processus de changement d'attitude des parents face à l'art abstrait, et ce, même si ces derniers n'y ont pas assisté.

Une mère exprime ainsi le crédit qu'elle accorde aux choix réalisés par l'enseignante de sa fille et se questionne en apprenant le thème de la visite suivie par sa classe - thème qu'elle pensait peu approprié pour des enfants de cet âge: "Je pense que probablement je me trompe, parce que si Mme F. est venue, c'est probablement parce que ce n'est pas ça, c'est que les enfants ont un autre accès " (mère de J., dix ans). Sa curiosité a donc été piquée en apprenant que sa fille avait apprécié cette visite scolaire. La confiance accordée par les parents à l'école en termes de culture est subjacente dans le discours des familles, à l'instar de ce couple de parents qui expliquent comment, alors qu'ils avaient cessé de visiter des musées à la naissance de leur fille craignant que les visites en famille soient des moments qu'ils qualifient eux-mêmes "d'horribles ", la première visite scolaire en maternelle de leur fille les a fait changer d'avis:

On a commencé [à visiter des musées en famille] grâce à l'école [...] Le fait que l'école l'amène, on s'est dit après tout, c'est solennel, mais c'est aussi adapté aux enfants. Franchement, oui, si l'école avait pas... moi j'aurais attendu qu'elle ait bien dix ans pour l'amener. Ou qu'elle en fasse la demande, qu'elle dise “oui, ça m'intéresse la peinture" " (mère de L., sept ans).

Pour une autre famille, qui ne visite que rarement les musées, l'école est véritablement le moyen d'y accéder en famille. Père et mère participent activement aux visites scolaires de leur fils " pour partager ça avec eux "; " sinon, on ne le ferait pas, ce qui nous manque, c'est la connaissance, c'est difficile d'amener les enfants quand on ne sait pas quoi leur dire " (père et mère de M., neuf ans).

\section{... à la visite en famille}

L'école et la pratique scolaire du musée semblent donc être pour certaines de ces familles non seulement un point d'entrée dans l'institution, mais aussi une manière d'appréhender différemment leurs visites familiales. En effet, à l'occasion de cette étude, une fois pénétré dans les salles dédiées au travail de Pierre Soulages et en voyant leur enfant évoluer et s'exprimer sur les œuvres, les adultes constatent que leur première intuition de l'art abstrait comme étant peu accessible en famille peut être nuancée. Les propos de cette mère sont révélateurs: «Je suis surprise qu'elle ait autant aimé [...] Qu'elle ait trouvé ces tableaux beaux, je pense qu'elle est sensible justement à ça, à ces tableaux. Je suis surprise " (mère de J., dix ans). Pour cette même famille, un changement d'attitude immédiat se produit: 
I: Pour une future visite de musée toutes les deux, vous aurez moins d'a priori pour aller voir des œuvres abstraites?

F: Moi je pense qu'on aura plus de plaisir à aller voir.

M: Plus de plaisir ou moins d'appréhensions, moins de petites, moins de réticences. Moins de euh, tu sais, de sentiments a priori un peu négatifs (mère et fille, J., dix ans).

On constate que le goût, les idées et les appréhensions des parents face au musée et à l'art abstrait constitueraient un frein important à la visite de ce type d'expositions en famille. Toutefois, la visite scolaire serait une manière pour des parents et leur enfant d'accéder à des activités auxquelles ils n'auraient pas pensé ou qu'ils se seraient en quelque sorte " interdites ». Par contre, à l'exception d'une mère, et contrairement à ce qu'avait constaté Marie Brûlé-Currie ${ }^{22}$, nous n'avons pas observé de changement dans les goûts des parents. Ce n'est pas le but de notre étude de questionner la relation esthétique des adultes à l'art abstrait, mais il est toutefois intéressant de noter que s'ils évoquent leur plaisir à écouter leur enfant exprimer leur attirance pour cette peinture, il ne s'agit pas pour les parents de changer ou de revoir leurs goûts personnels. Il ressort toutefois de ces entretiens l'idée forte que le fait de visiter en famille et après une visite scolaire a un réel impact sur la qualité de visite et d'observation des adultes.

\section{Observer en famille des œuvres d'art abs-} trait après une visite scolaire: quels impacts sur l'expérience de visite des parents?

\section{Le rapport aux ouvres}

La visite en famille après une visite scolaire offrirait une certaine qualité de visite que les adultes semblent particulièrement apprécier. Les entretiens mettent en évidence le fait que plusieurs parents considèrent que si on leur avait été demandé de visiter ces espaces sans leur enfant, leur visite aurait été plus rapide, moins attentive et moins riche.

Un temps de visite ressenti comme plus important À l'exception d'une mère, les parents de notre étude estiment tous qu'ils auraient passé moins de temps dans ces espaces si leur enfant n'avait pas suivi une visite scolaire au préalable, ou s'ils avaient été seuls. "Je ne m'y serais pas attardée [...] On a passé beaucoup plus de temps que si j’y étais allée toute seule " (mère de L., sept ans). "J'aurais été plus rapide dans Soulages, voilà, j'aurais été plus rapide, mais par contre avec R., on a été plus lents vers Soulages, parce qu'il voulait m'expliquer ce qu'il avait fait avec l'école " (mère de R., sept ans). « Toute seule, sans M., sans explication, je serais passée plus vite " (mère de M., neuf ans). Pourtant, si nous calculons le temps passé dans les salles et que nous le comparons au temps passé par un échantillon similaire de familles dont l'enfant n'a pas suivi de visite scolaire au préalable, nous constatons qu'en moyenne les familles ont passé un peu plus de 14 minutes dans les salles Soulages, ce qui équivaut à notre échantillon de familles " témoins ". Cependant, les parents que nous avons interrogés ont le sentiment d'avoir pris davantage leur temps. Ce sentiment pourrait être attribuable à l'attention toute particulière que certains d'entre eux expliquent avoir portée aux œuvres en compagnie de leur enfant.

\section{Une observation ressentie comme plus fine, plus attentive}

La présence de l'enfant et les échanges induits par la visite en famille semblent en effet engendrer une certaine qualité d'observation de la part des parents, qui porteraient davantage attention aux œuvres. Par exemple, cette mère qui avait visité seule il y a quelque temps cette partie du musée sans faire " trop d'efforts " explique comment la présence de son fils l'a fait changer d'attitude de visite: 
J'avais moins fait attention $[\ldots]$ Très vite je n'ai pas adhéré et je n'ai pas trop fait d'efforts. Le fait que je sois là avec $\mathrm{H}$., le fait qu'il l'ait vue avant, j’y ai attaché plus d'attention [...] Oui, oui, c'est sûr [...] J'ai écouté ce qu'il m'a dit et j'ai regardé en fonction. Enfin voilà, c'était un échange, donc l'attention n'est pas la même (mère de H., dix ans).

Par ailleurs, les parents indiquent que leur regard a été non seulement plus attentif, mais aussi plus précis, sous l'influence de leur enfant. Les parents expliquent ainsi avoir remarqué des aspects de l'œuvre de Soulages qui leur auraient échappé s'ils avaient visité seuls : "J'aurais pas vu tout ce que j'ai vu là. J'ai vu plus de choses " (mère de L., sept ans). Les effets de lumières caractéristiques de l'œuvre de Soulages sont notamment cités plusieurs fois. Dans cet échange, nous notons clairement l'influence de la présence de l'enfant et de la visite scolaire sur les observations de l'adulte.

I: Vous me disiez qu'il vous avait montré la lumière?

M: Oui oui.

Fs: C'est les traits, les effets de lumière, ça fait les traits. Au début quand je suis venu avec l'école, j'étais impressionné parce qu'il y avait les traits.

\section{I: C'est quelque chose que vous auriez remarqué?}

M: Non, ça c'est un truc auquel je n'aurais pas fait attention (mère et fils, R., sept ans)

Pendant la visite, six des enfants ont demandé à leurs parents d'observer les œuvres selon différents points de vue, comme cela leur avait été proposé pendant la visite scolaire. En leur indiquant où et comment se placer par rapport à l'œuvre, ils les ont amenés à observer les jeux de lumière sur la matière:

F: Attends, mets-toi un peu plus sur la droite. Regarde, tu vois? Tu ne vois pas la même chose. Regarde là, et tu bouges, et tu vois pas la même chose.

M: D'accord. C'est vrai

F: Viens maman, aussi là tu vois. Regarde, regarde-le en te levant, regarde-le.

Regarde, regarde. T'as vu, il bouge!

M: Ah oui, dis donc.

F: Regarde, là il est de plus en plus foncé, et mets-toi comme ça, tu vois? (mère et fille, J., dix ans).

Il semblerait donc que la présence de l'enfant et son expérience préalable de visite scolaire aient un impact assez important sur l'expérience de visite des adultes. Le rôle de l'enfant pendant ces visites est donc à questionner. S'il semble apprendre des éléments relatifs à l'œuvre de Soulages à ses parents en leur restituant une partie du contenu de la visite, peut-on considérer que l'enfant endosserait un rôle de médiateur pendant cette visite?

\section{Visiter en famille après une visite scolaire: ou l'adulte déchargé de sa fonction de "parent-guide "}

\section{Des habitudes de visite révélatrices d'un schéma de transmission verticale}

Les huit familles rencontrées ont déjà visité au moins une fois un musée d'art ensemble. L'entretien mené avec elles nous a permis de connaître en partie la manière dont se déroulent d'habitude leurs visites de musée. On constate que chaque parent, à sa manière, développe ce que nous pourrions qualifier de "stratégie de visite ". Une mère souligne par exemple la difficulté qu'elle peut ressentir face aux questions de ses enfants: "J'essaie d'expliquer, des fois ils me posent des colles, je ne sais pas répondre " et explique comment elle détourne cette difficulté en l'anticipant: "Je crois qu'il faut se préparer, enfin, les thèmes, parce que tout est expliqué sur le site du musée, les thèmes, les salles " (mère de 
R., sept ans). Une autre souligne avec plus de force ses appréhensions : «Imaginez, nous on vient en famille, on ne connaît rien sur les peintres, il n'y a pas de thématique précise, on n'organise rien de spécial. Je me dis qu'au lieu de les sensibiliser au musée, je vais les en dégoûter. Quand on est pas des spécialistes, c'est très difficile. " Malgré le coût des visites guidées, c'est, explique-t-elle, la solution qu'elle a trouvée pour visiter en famille. Les propos de ces mères soulignent leurs appréhensions quant à la visite en famille. Ils sont l'illustration des travaux de Robert Lakota ${ }^{23}$ qui a montré que les adultes accompagnés d'enfants ont tendance à choisir des thématiques de visite qui leur sont familières et dont ils maîtrisent le contenu. Ces "préparations " qui sont ici évoquées montrent que le musée est reconnu par les adultes comme un lieu de transmission et que ces derniers se sentent investis d'une réelle " mission " d'éducation. D'autres parents interrogés développent et imaginent des activités pour impliquer leur enfant dans la visite. Que ce soit sous la forme de questions / réponses questions, lui dire "et là qu'est-ce que tu vois? Est-ce que ça te plaît?" Voilà, sinon, elle va être totalement passive et attendre que ça se passe [rires] " (mère de L., sept ans) -; de petits jeux - «On faisait des jeux, en fait, on rentrait dans une salle et c'était le premier qui trouvait une vache. Ou bien, celui qui avait gagné, c'est celui qui avait vu le plus de chiens " (mère de J., dix ans); « En entrant dans un musée je leur fais un petit défi: le premier à trouver un lapin choisit le goûter " (mère de I., huit ans) - ; ou d'activités telles que des dessins:

M: J'avais prévu quand même un petit calepin et des crayons, et il était bien, on avait passé deux heures dans le musée. On était passés de salle en salle. Et de temps en temps il s'intéressait, de temps en temps il dessinait ses trucs.

I: Et vous aviez eu l'idée d'amener de quoi dessiner?

23 LAKOTA, Robert. The National Museum of Natural History as a Behavioral Environment. Washington, DC: Smithsonian Institution, 1975; et JONCHERY, Quand la famille vient au musée..., op. cit., p. 103.

M: Oui, mais quand vous gérez des enfants en bas âge, on pense à ces trucs-là. Parce que moi je venais pour lui, pour l'amener, mais aussi pour moi, me faire plaisir. Donc je savais bien que la notion du temps pour moi était pas la même que pour lui. Donc c'est un peu comme toutes les sorties, on sait prévoir, on s'organise (mère de H., dix ans).

Ces stratégies, pensées à l'avance ou improvisées, permettent non seulement d'allier le plaisir de l'adulte et celui de l'enfant pour une activité qu'il n'a visiblement pas choisie, mais placent clairement les adultes dans une attitude active, voire participative, de visite : ils mènent la visite et se positionnent en médiateur du musée auprès de leur enfant.

En partant de ce constat, les entretiens ont permis de faire ressortir en quoi et comment les relations parents-enfants ont pu être différentes lors de cette visite des salles Soulages après une visite scolaire de l'enfant.

\section{La visite en famille après une visite scolaire: l'inversion des rôles?}

Les adultes retiennent de cette visite sa singularité, induite par la visite scolaire que leur enfant a suivie les semaines précédentes. Ils estiment non seulement avoir appris de leur enfant, mais lui reconnaissent volontiers un statut qui pourrait s'apparenter à celui de connaisseur, ou de "déjà initié " et, par là même, certains d'entre eux semblent apprécier d'être en quelque sorte "déchargés " de leur statut habituel de parent-meneur. L'expression « inversion des rôles " est ainsi employée à plusieurs reprises par les familles.

\section{Le parent en situation d'apprenant}

Les parents partagent tous en effet le sentiment d'avoir appris des choses de leur enfant. 
M: Il y avait plein de choses que je ne savais pas sur sa manière de faire, qui, c'était, les couleurs, tout ça. Moi je trouvais que c'était un peu triste tout ça, et toi?

F: Non.

M: Et bien, moi j'aurais appris moins de choses [si j'avais visité seule] Parce que tu m'as appris plein de choses sur Pierre Soulages (mère et fille, L., sept ans).

M: Il m'a dit qu'avec sa femme ils ont donné les tableaux à Montpellier.

Fs: Au musée, comme ils s'étaient rencontrés ici, dans ce musée.

M: Parce qu'ils se sont rencontrés ici dans ce musée, c'est ce qu'il m'a dit. Donc il m'a appris des choses (mère et fils, R., sept ans).

L'écoute des conversations pendant la visite révèle que les paroles des enfants font en effet largement écho à celles de la médiatrice qui les a accompagnés lors de leur visite scolaire. C'est semble-t-il très naturellement qu'ils retransmettent à leurs parents les connaissances acquises sur l'artiste à cette occasion: « Elle a transmis tout ce qu'elle avait appris pendant la visite " (mère de P., dix ans). Les aspects liés à la vie du peintre, mais aussi sa technique de création, son affection toute particulière pour révéler la lumière et les relations privilégiées que Pierre Soulages entretient avec le musée Fabre, sont autant de thèmes qui ressortent et reviennent dans la parole des enfants. Par ailleurs, certains enfants mettent aussi un point d'honneur à réutiliser un vocabulaire nouveau et à l'expliquer à leurs parents, par exemple "polyptyques" ou " monochromes". Que ce soit en termes d'informations retransmises verbalement ou dans l'attitude adoptée par les enfants - par exemple diriger le regard du parent selon plusieurs points de vue pour apprécier au mieux les effets de la lumière sur la matière, comme cela leur avait été suggéré par la médiatrice lors de la visite scolaire -, nous pouvons dire que les enfants rencontrés adoptent une attitude de visite active.

Ce statut est d'ailleurs souligné par six parents qui, lors de l'entretien, reconnaissent à leur enfant la qualité de "guide " pendant cette visite: "C’est lui qui nous a guidés, il était vraiment dans son rôle, vraiment passionné " (mère de M., neuf ans). Cette inversion des rôles semble être appréciée par les parents qui sont aussi surpris: "Je ne m'attendais pas à ce qu'elle démarre la visite comme ça! C'était surprenant, elle s'est donné cette fonction de guide " (mère de P., dix ans).

Quant aux adultes pour qui le travail de Soulages n'était pas familier ou qui le découvraient, le fait d'apprendre et de bénéficier d'informations, par le biais de leur enfant, est appréciable: «Elle m’a expliqué un peu. Et c'était vraiment très bien, très intéressant, j'ai appris des trucs [...] Il faut que tu y ailles plus souvent au musée avec l'école, puis après tu me fais guide! Tu vas devenir guide, c'est pas mal, comme métier! » (mère de L., sept ans). Pour une mère qui connaissait le travail de Soulages au préalable, et qui avait déjà visité ces salles, le fait d'apprendre, ou plutôt de "réapprendre ", de la bouche de sa fille donne à l'information une valeur toute particulière: "Je le sais, comment dire, parce que je l'ai lu, maintenant je le saurai parce que ma fille me l'aura appris, c'est pas la même chose " (mère de J., dix ans).

Le caractère exceptionnel de la situation de transmission et d'apprentissage est ainsi relevé de manière claire par deux mères : "C'est déjà beaucoup l'idée de se dire qu'elle va apprendre, elle va transmettre quelque chose qui normalement ne se transmet pas dans ce sens-là. D'habitude c'est toujours les grands qui apprennent aux petits. Et sur des choses, finalement, peut-être subjectives, sur des sentiments, sur des impressions, c'est l'enfant qui va transmettre à l'adulte, c'est important pour un enfant " (mère de J., dix ans). "Des fois c'est pas que le parent qui apprend des choses aux enfants, des fois c'est les enfants qui 
apprennent aux parents [...] ça m’a fait plaisir, pour une fois que voilà, les rôles se sont inversés et c'était sympa " (mère de R., sept ans).

Par ailleurs, les propos de cette mère reflètent ce qui pourrait être interprété comme le plaisir d'être libérée de son statut de " parent " à l'occasion de cette visite: « Là c'était elle qui était guide. Ce n'est pas à moi de présenter un tableau. Non, non, là c'est [elle] qui me, comment dire, qui m'apprend. C'est bien " (mère de J., dix ans).

\section{Un sentiment de fierté d'apprendre de son enfant}

Il ressort de manière générale des entretiens un sentiment de fierté ressenti par les parents qui viennent de partager avec leur enfant l'observation des œuvres de Pierre Soulages. Une mère explique à plusieurs reprises dans l'entretien combien son fils l'a "impressionnée ", tandis que d'autres parents sont surpris de l'atvisite scolaire en retenant "plein de choses".

M: j'étais surprise de voir tout ce qu'elle avait retenu, ou savait, je ne sais pas après d'où vient ce qu'elle me disait. Parce que je trouvais qu'elle avait vraiment une bonne connaissance et elle m'a surprise parce qu'elle ne regardait pas que l'œuvre, mais l'œuvre dans le musée. L'éclairage... quand même, c'est pas mal [rires]! Hein? L'éclairage, tu m'as parlé des fenêtres qui apportaient la lumière aux tableaux, que c'était justement pour lui que ça avait été mis (mère de L., sept ans).

Si le statut de « guide » est mis en valeur et souligné par les parents lors de l'entretien, sur les huit enfants rencontrés, six ne semblent pas conscients d'avoir tenu ce rôle auprès de leur(s) parents(s). Seuls deux enfants l'assument pleinement et semblent en retenir une certaine fierté: "J'étais une sorte de guide " (M., neuf ans); «C'est comme si je travaillais ici! [rires] ” (R., sept ans).
Ces éléments issus des propos des huit familles interviewées viennent alimenter l'hypothèse de départ supposant que l'enfant, en passant du statut d'élève-visiteur à celui de fils / fille, endosserait auprès de ses parents le rôle de guide du musée et des œuvres. Par ailleurs, deux familles soulignent la réelle différence de relation entre leur visite des salles Soulages et la suite de leur déambulation dans le musée face à des œuvres que les enfants n'avaient pas découvertes lors de la visite scolaire. "La preuve c'est qu'après on a déambulé dans d'autres salles, et c'était beaucoup moins cohérent, j'essayais de construire quelque chose, mais voilà... " (mère de P., dix ans). "Quand on était dans Soulages, c'était beaucoup elle qui parlait, qui s'exprimait. Et quand on était devant d'autres tableaux par la suite, c'était plus moi qui la relançais et qui essayais de la sensibiliser " (mère de A., huit ans). Ces propos montrent très clairement l'influence de la visite scolaire sur le rôle et le statut du parent et de l'enfant lors de la visite en famille: une fois sortis des salles connues par les enfants, les parents reprennent leur rôle de médiateur.

\section{Discussion}

Cet article propose l'analyse qualitative des comportements de visite d'un petit nombre de familles participant à une étude en cours portant sur un nombre plus important de participants. L'échantillon réduit de huit familles a été choisi pour les similitudes de leurs profils de familiarité muséale et culturelle. Rappelons aussi que les familles sont invitées à revenir visiter le musée avec leur enfant et que les premières familles ayant répondu à l'invitation sont composées 1) d'enfants ayant apprécié la visite scolaire puisqu'ils souhaitent y revenir avec leurs parents, 2) de parents sensibles à notre démarche de recherche et / ou à la pratique de la visite de musée en famille. Les habitudes de visite de ces familles, relevant de réelles stratégies de médiation parentale, témoignent bien de leur appétence pour les activités culturelles en famille. Nous pouvons donc penser qu'elles ne sont pas représentatives de l'attitude des familles en général et notamment des familles "non-publics». Les processus de changement 
d'attitude et de situation de transmission ici évoqués seront sans doute révélateurs des caractéristiques d'une certaine catégorie de familles et seront, nous le pensons, l'un des résultats parmi d'autres que l'étude, une fois terminée, révélera.

Par ailleurs, il nous semble nécessaire d'interroger le mode de recueil de ces propos: la présence de l'interviewer, mais aussi de l'enfant, peut, à notre avis, pousser l'adulte à exprimer de manière plus importante un sentiment de fierté à l'égard de son enfant, en accentuant le rôle que ce dernier a réellement tenu pendant la visite. Nous souhaitons à ce sujet proposer un moyen de compléter nos données recueillies en entretien afin d'en enrichir l'analyse. Afin de nous rendre compte de la manière dont parents et enfants interagissent pendant leur visite, nous avons retranscrit les conversations de huit familles (quatre dont l'enfant est venu en visite scolaire et quatre dont l'enfant venait pour la première fois au musée). Nous les avons étudiées à l'aide d'un logiciel d'analyse de discours afin de repérer les tendances relatives aux modes de communication et de transmission adoptés par les adultes et par les enfants. Les propos tenus par les enfants et les parents ont été retranscrits et traités séparément, c'est-àdire comme des corpus automnes du point de vue de l'instance énonciatrice. Nous ne donnons ici qu'un échantillon des analyses possibles avec cette méthode comparative. Ainsi, si nous nous focalisons sur les trois marqueurs énonciatifs suivants: 1) l'utilisation des verbes " regarder " et "voir "; 2) l'utilisation des pronoms " tu » et " je »; 3) le nombre de mots prononcés par chacun des interlocuteurs, il s'avère que ces marqueurs strictement linguistiques fonctionnent comme des révélateurs d'une posture pédagogique adoptée par le locuteur.

\section{Le recours aux formules du type " regarde", " tu vois"}

Si les différences sont minimes, nous pouvons toutefois noter que l'enfant ayant suivi une visite scolaire aura plus tendance à impliquer son ou ses parents en les invitant sensiblement plus à « regarder " et à " voir ». Du côté des parents, il semble que lorsque leur enfant a suivi la visite scolaire, ils aient moins tendance à accompagner leur regard, à les interpeller. Par contre, quand l'enfant n'a pas suivi la visite scolaire, les parents se positionnent davantage en "médiateurs ", encourageant leur enfant à regarder, et accompagnant leur regard.

\section{Le recours aux pronoms " je " et " tu "}

S'intéresser aux pronoms utilisés lors de la visite nous permet de déterminer qui, du parent ou de l'enfant, est au centre de la conversation. Nous ne notons pas de différence entre les deux échantillons en ce qui concerne la place prédominante que les enfants semblent occuper dans les conversations. Nous relevonspar ailleurs une tendance des enfants à l' "égocentrisme " (chose qu'avait notée BrûléCurrie lors d'une étude menée auprès de jeunes enfants et de leur maman en visite $\left.{ }^{24}\right)$. Ceux-ci s'expriment plus à la première personne que leurs parents ( «je ") et s'adressent moins à eux (" tu »). Si de tels chiffres peuvent difficilement être révélateurs sur un nombre restreint de familles, il est toutefois intéressant de relever que l'utilisation de la première personne du singulier est moins fréquente de la part des enfants ayant participé à une visite scolaire. Ce dernier point pourrait laisser penser qu'ils ont plus tendance que les autres à s'adresser à leurs parents.

\section{La richesse des énoncés échangés en nombre de mots}

Enfin, les outils informatiques nous permettent objectivement de connaître qui, de l'enfant ou du parent, s'exprime le plus devant les œuvres. De manière générale dans nos corpus, les enfants prennent plus souvent la parole que les adultes. De plus, nous remarquons que la différence de prise de parole parent / enfant est beaucoup plus nette lorsque ce dernier a visité le musée au préalable avec l'école. 


\section{Conclusion}

Il est d'ores et déjà intéressant de relever les changements d'attitudes et de comportements des adultes observés au cours de la visite familiale. Il apparaît que la visite scolaire a un réel impact sur les modes de communication et le statut que parents et enfants adoptent lors d'une visite en famille. Ces éléments sont des pistes de travail pour creuser le rôle de la visite scolaire dans les attitudes de transmission des visiteurs en famille et pour interroger un hypothétique modèle de transmission ascendante. En allant dans le sens de De Singly et Octobre, le modèle de transmission verticale reste en effet à réinterroger. La transmission ascendante serait rendue possible par le sens particulier que revêt la transmission moderne en produisant un héritage qui laisse une large initiative aux héritiers ${ }^{25}$. En prenant parfois en compte le désir de l'enfant et en lui conférant un rôle moteur dans l'acte de visite, l'adulte et l'enfant se retrouveraient dans des rôles de transmission quasi inversés qui suggèrent une rupture avec la logique traditionnelle de la transmission. « Les parents, n'étant pas toujours des guides, doivent rendre visible cette rupture avec la définition du parent pédagogue, du parent professeur, du parent transmetteur. ${ }^{26}$ " La pratique scolaire de l'enfant serait-elle la solution pour réinterroger ce modèle et alors revoir les logiques et les motivations des visiteurs des musées d'art? 


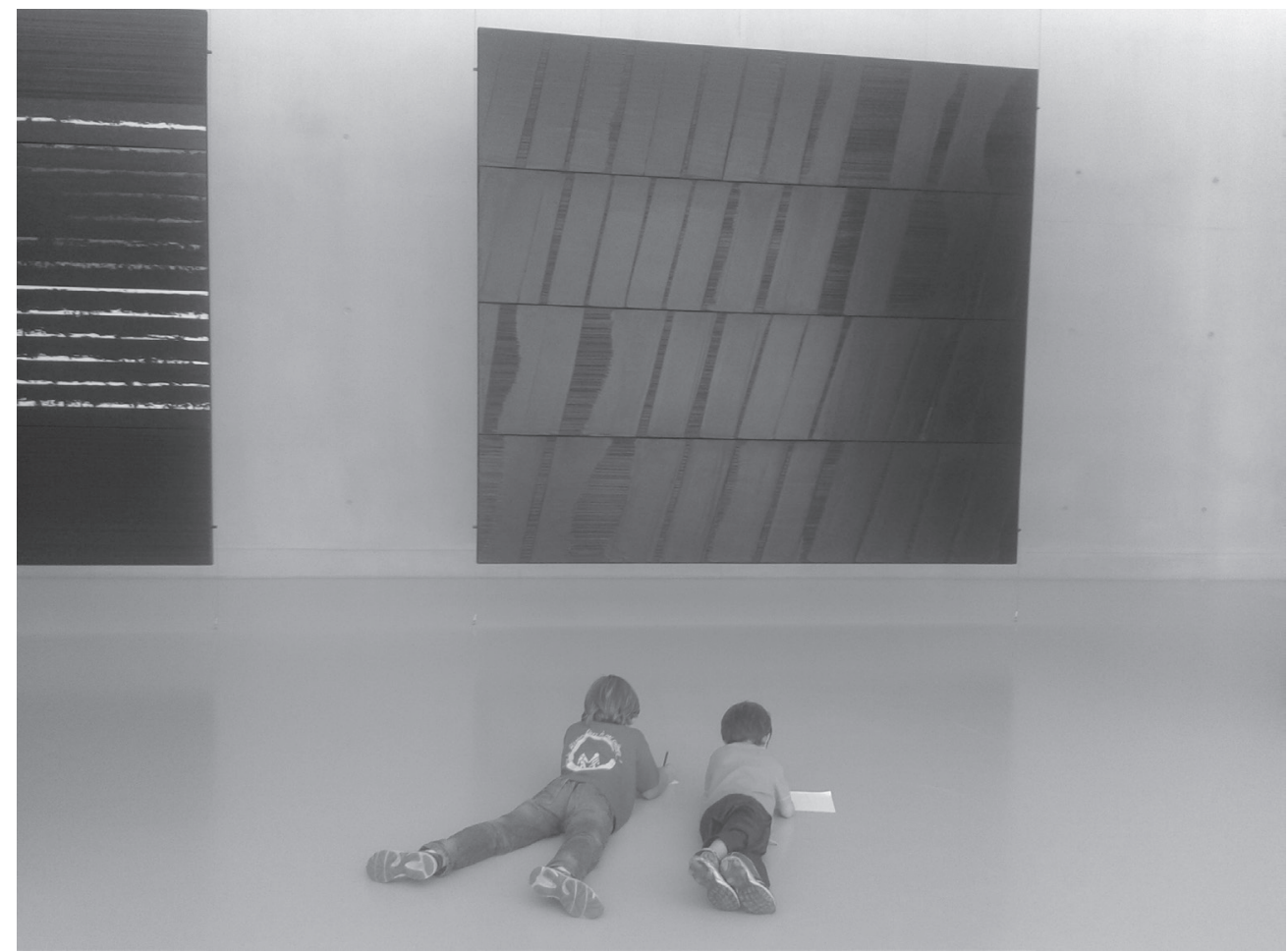

Dessin dans les salles Pierre Soulages, Musée Fabre de Montpellier Agglomération [Aude Joly]. 
Aude Joly \& Marie-Sylvie Polie, translated by Allana Carlyle

\section{Discovering the works of Pierre Soulages as a Family After a School Visit}

Over the last few years we have witnessed the emergence of a new form of communication from museums, one that increasingly addresses a new category of visitors: Families. The terms "family visit", and "for families" are used in a general sense on internet sites and in programs for museum cultural activities, notably in art museums. In addressing the family as a unit, museums place emphasis on the social aspect of the museum visit. By considering the family as general public, the museum takes into account the visitors in relation to the art work and to the exhibit but also the visitors' relationships with each other. But what is the experience of visiting a museum as a family? What happens when an adult and child observe art together?

This article, based on research in progress for a Doctorate in Museology, will examine the way parents and children interact with each other when faced with a work of art, and more specifically a contemporary work of art, while visiting an art museum together. What do they say to each other? What roles and methods of communication do they adopt?

To answer these questions, a field study was conducted at the musée Fabre in Montpellier. Participating families were comprised of an elementary school-aged child, of his/her parents, as well as any siblings. The methodology was to record their conversations with a lapel microphone as they visited the second level of the museum freely, autonomously, and at their own pace. The visit was followed by a semi-structured interview. This article focuses on the family interactions in the galleries dedicated to contemporary artist Pierre Soulages, where the participating families were invited to begin their museum visit.

The sample of participating families was deliberately not a reflection of the general public visiting the musée Fabre. Participating families were invited to visit the museum specifically as part of the framework for this study. The participants had a range of museum experiences, however they all shared the distinctive feature of visiting the musée Fabre for the first time together as a family.

In addition, some children from the sample families previously participated with their school classes in a guided visit of the Soulages galleries while other children were visiting the museum for the first time. By introducing the school visit as a variable prior to the family visit, this study will examine the role of the child in his/her family's relationship with the museum and with contemporary art. Will the school visit act as a link between the museum and the family? In shifting from the role of a student visiting the museum to the role of a son or daughter participating in a family visit to the museum, will the child take on the role of museum interpreter for their parents? 
Research conducted in France and Quebec suggests that for an adult who is unfamiliar with art museums, the fact of being accompanied by their child leads them to view the art museum with new eyes ${ }^{1}$ and that being in a family context can provide reassurance for adults unfamiliar with museums ${ }^{2}$. This article will present the hypothesis that children, by legitimizing their presence and assuming a didactic role, can be seen as interpreters of the contemporary art museum for their parents.

1 BRULE-CURRIE, Marie. "When three-to-five-yearsolds and adults look at modern and abstract art together $\%$. In. ALLARD, Michel et Bernard LEFABRE (dir.) Le musée, un projet éducatif. Montréal: Éditions Logiques, 1996, p. 109-125. 2 JONCHERY, Anne (2005). Quand la famille vient au musée: des pratiques de visites aux logiques culturelles. (Doctoral thesis). National Museum of Natural History. 